\title{
ILCEA
}

Revue de l'Institut des langues et cultures

d'Europe, Amérique, Afrique, Asie et Australie

16 | 2012

La culture progressiste à l'époque de la guerre froide

\section{À propos de notre film Les Enfants de Golzow}

Barbara Junge et Winfried Junge

Traducteur : François Genton

\section{CpenEdition}

\section{Journals}

Édition électronique

URL : http://journals.openedition.org/ilcea/1253

DOI : 10.4000/ilcea.1253

ISSN : 2101-0609

Éditeur

UGA Éditions/Université Grenoble Alpes

Édition imprimée

ISBN : 978-2-84310-232-5

ISSN : 1639-6073

Référence électronique

Barbara Junge et Winfried Junge, "À propos de notre film Les Enfants de Golzow », ILCEA [En ligne], 16 | 2012, mis en ligne le 04 juillet 2012, consulté le 22 mars 2021. URL : http://journals.openedition.org/ ilcea/1253 ; DOI : https://doi.org/10.4000/ilcea.1253

Ce document a été généré automatiquement le 22 mars 2021.

(C) ILCEA 


\title{
À propos de notre film Les Enfants de Golzow
}

\author{
Barbara Junge et Winfried Junge
}

Traduction : François Genton

1 Vous avez pu découvrir hier un court extrait ${ }^{1}$, un «échantillon » de notre travail. Il s'agit d'une observation de longue durée, la plus vieille de l'histoire du cinéma, des Enfants de Golzow, qui firent le $1^{\mathrm{er}}$ septembre 1961 leur entrée dans l'école primaire d'un village situé au bord de l'Oder au nord de Francfort sur l'Oder, dans l'ancienne République démocratique allemande.

2 Jusqu'en 2007 nous avons suivi leur parcours dans la vie, à travers les vicissitudes de la vie, en 19 films. Le premier durait douze minutes et demi, le dernier, en quatre parties, neuf heures et demi.

3 Le film à l'origine le plus long fut en 1980/1981 Lignes de vie dont les deux parties duraient 4 heures et 17 minutes.

4 C'était le $8^{\mathrm{e}}$ film et celui qui remporta le plus grand succès parmi ceux qui furent tournés en RDA. Il est en partie la raison de la survie du projet en RDA et de sa poursuite, avec dix films supplémentaires, en RFA.

5 À l'issue d'un sondage international entrepris par la fondation de la cinémathèque allemande, Lignes de vie figura en 1995 sur la liste des 100 films allemands les plus importants du premier siècle de l'histoire du cinéma.

6 Lignes de vie présente les portraits particuliers de neuf enfants et en esquisse cinq supplémentaires dans l'épilogue. Au total 14 élèves choisis parmi les 26 du cours préparatoire de 1961.

7 Vous n'aurez vu hier que trois de ces neuf portraits, seulement le tiers d'un film qui ne peut emporter l'adhésion que dans son intégralité. Nous vous prions d'en tenir compte.

Les DVD des 19 films sont désormais pourvus de sous-titres anglais. 

raison principale est le sujet du colloque : «La culture progressiste durant la guerre froide ». En quoi Lignes de vie pourrait illustrer cette culture, c'est à la discussion de le montrer.

11 Le film a désormais plus de trente ans. Il est donc depuis longtemps dépassé par la vie des enfants de Golzow. Si nous en avions le temps, nous n'aurions aucune difficulté à raconter la suite de leur vie.

\section{Lignes de vie s'arrête, décrivent trois nouvelles décennies, durent plus de deux heures} chacun.

Heureusement - on peut le voir ainsi - le sujet de ce colloque limite notre choix à la période 1961-1980. Ce sont des années pendant lesquelles on pouvait encore fonder un certain espoir sur l'autre État allemand, mais qui laissent déjà percevoir la stagnation à venir, même si l'on ne prévoyait pas l'effondrement de l'automne 1989.

Depuis plus de vingt ans la division de l'Allemagne relève du passé. La jeunesse d'aujourd'hui est née en RFA. Faut-il pour autant tout ignorer de la RDA?

Il y a quelque temps on m'a demandé de parler à la jeunesse. Comme il s'agissait d'un discours destiné aussi aux adultes, je veux le répéter ici, dans une version à la fois abrégée et complétée.

Les « enfants de Golzow » n'ont plus seulement des enfants, certains d'entre eux ont des petits-enfants, sont grand-père et grand-mère.

Et ils vivent avec leurs enfants et petits-enfants parmi nous.

Dans un État, qui s'appelle République fédérale d'Allemagne.

Qui s'appela un jour République démocratique allemande, en abrégé RDA.

dans un pays qui fut toujours l'Allemagne.

Que disent-ils de la RDA à leurs petits-enfants,

un pays qui appartenait au passé

avant même le jour de leur naissance?

Que dirons-nous, les cinéastes, 
la génération des arrière-grands-parents,

de cette autre République allemande?

Seulement qu'elle n'était pas aussi démocratique

que le nom qu'elle s'était donné ?

21 Moi aussi, bien sûr, j'ai été un enfant.

Jusqu'à l'âge de dix ans, mon enfance

fut une enfance sous Hitler.

À ne pas confondre avec Honecker.

Mais à quoi bon cette comparaison

quand les enfants d'aujourd'hui

ne connaissent peut-être ni l'un ni l'autre?

Nos tout petits peuvent sans doute ignorer cela,

mais avec quelques années de plus un tel savoir ne peut nuire.

22 Mon enfance

fut une enfance sans père,

car quand j'avais quatre ans

il dut partir à la guerre

dont il n'est pas revenu.

Mon enfance,

ce fut les bombes,

la course vers l'abri.

Ce fut l'angoisse et parfois la joie :

la joie toute simple de vivre un nouveau matin,

de trouver sur le chemin de l'école et de pouvoir exhiber

un bel éclat de l'obus

tiré la nuit par la défense anti-aérienne.

La guerre n'avait pas seulement lieu là

où mon père était allé.

Elle s'abattait aussi sur ma mère et moi.

Elle pouvait arriver

lorsque l'école venait de commencer.

Quand les sirènes retentissaient sur les toits,

parce qu'à nouveau « une escadrille de bombardiers ennemis ",

comme disait la radio,

s'approchait de Berlin et que nous étions vite renvoyés chez nous.

Si j'étais sur le point de subir

la canne du maître,

c'était le salut,

mais sinon?

25 J'arrête.

J'aime mieux parler de la paix,

qui vint avec le printemps de l'an 1945 et qui,

en compagnie de tous ceux qui avaient survécu à la guerre,

me fit retourner à l'école pour apprendre. 


\section{qu'on nous avait confié à cet effet}

c'était la RDA.

Dans ce tiers d'Allemagne

qui était devenu, après la division

de l'Allemagne par les quatre puissances victorieuses, zone d'occupation de l'Union soviétique

- de la Russie, comme on dit simplement aujourd'hui -

le pouvoir ne devait

jamais retomber entre les mains de ceux

qui avaient causé deux guerres mondiales

dont ils avaient tiré profit.

29 Sous la protection de la puissance victorieuse, qui

avec vingt millions de morts

avait accompli le plus grand sacrifice de la dernière guerre,

bientôt protégée aussi par ses propres forces armées,

alliées à celles d'autres pays européens

qui voulaient eux aussi devenir socialistes

et, en fin de compte, derrière un mur

se réalisait l'expérience

qui consistait à fonder une démocratie

qui devait vraiment signifier " pouvoir populaire »

pour contribuer à empêcher

que pour la troisième fois se répète une guerre,

qui pouvait signifier la fin du monde,

étant donné l'existence d'arsenaux nucléaires.

Ou comme Bert Brecht,

un poète qui avait choisi de vivre dans cette RDA,

avait dit de la "grande Carthage ",

qui mena trois guerres:

«Elle était encore puissante après la première,

encore habitable après la deuxième,

elle était devenue introuvable après la troisième. »

31 Était-ce un projet injuste

que de vouloir créer une Allemagne différente de l'ancienne avec tous ses crimes?

Mais lors d'une expérience inévitablement 
on commet des erreurs

et l'on se heurte à l'incompréhension, au scepticisme, au soupçon

et l'on suscite des adversaires.

\section{Il faut d'abord savoir qu'elle a existé.}

c'était quoi la RDA?

C'est à un tel savoir

que veut d'abord contribuer notre film d'observation de longue durée sur les « enfants de Golzow ».

Plus exactement :

Il veut contribuer à une vision nuancée, à un jugement historique juste.

Barbara et moi pensons ici à Theodor Fontane,

le poète du paysage brandebourgeois, et à sa phrase intelligente :

«L'Histoire traite de morts

qui ne peuvent pas se défendre.

Raison de plus pour leur rendre justice. »

Si l'on me demande

ce que fut la RDA,

alors je réponds d'abord simplement ceci :

"J'ai pu y vivre. »

Et j'ai pu y faire des films durant presque 5 décennies,

Pour lesquels il n'y avait

- me dit-on -

rien de comparable dans l'ancienne RFA.

Si l'on demande à d'autres,

ils diront peut-être

"Je ne pourrais pas y vivre."

Ou bien : 
"J'ai pu y vivre,

puis je n'ai plus voulu

parce que je voulais faire autre chose de ma vie. »

Pour eux, le Mur - ce « rempart antifasciste »-

était un rempart

avant tout érigé contre eux-mêmes

pour les empêcher

de découvrir le monde

et de décider eux-mêmes

de leur lieu de vie et de travail,

de leur avenir professionnel et personnel.

La RDA

qui voulait construire le socialisme

avait besoin de chacun de ses citoyens,

en particulier de ses jeunes.

Elle voulait gagner leur soutien,

Ne serait-ce que pour ce but politique.

Elle voulait les conquérir par l'éducation

et finit par les perdre d'innombrables fois

dès qu'ils prenaient conscience de

ce qui dans le «Roi des Aulnes » de Goethe prononce ces paroles :

"Si tu n'es pas consentant, j'userai de la force. »

Ce qui était et aurait dû rester une offre

devint une offre sans autre choix :

le socialisme (Sozialismus) -

dans certains cahiers d'école on écrivait, dit-on, Sozialismu $\beta^{3}$, le socialisme forcé.

« La cause » voulait que l'on crût nécessaire et possible

d'instaurer un pouvoir populaire sans démocratie.

Le socialisme en tant que loi universelle :

Erich Honecker formula imprudemment cette sentence proverbiale :

«Le socialisme dans son cours victorieux

Avance n'en déplaise aux ânes et aux bœufs. »

Ce ne sont certainement pas ces derniers qui ont interrompu son cours,

et il reviendra à nos petits-enfants

de déterminer

les causes ou les agents de son échec.

En théorie la RDA pouvait se croire

en avance d'une époque sur la RFA.

Cela pouvait inciter à la fierté,

mais cela incitait aussi à l'insouciance.

J'emprunte encore un mot à Brecht :

"Le Progrès ", disait-il,

« ce n'est pas avoir des idées progressistes, mais progresser soi-même.

Le Progrès, c'est

ce qui permet de progresser ou y contraint. »

Et cette sentence, on aurait dû l'inscrire

dans le cahier des charges de l'expérience RDA, mais aussi ailleurs. 
Elle garde sa validité

aujourd'hui aussi et dans tous nos pays.

Que cela soit dit à tous ceux

qui se satisfont déjà

du monde tel qu'il est

comme du « meilleur des mondes possibles ».

La RDA - l'État

que les pères, les grands-pères, les arrière-grands-pères

fondèrent, construisirent... et abandonnèrent -

avait beaucoup de visages

et n'était pas cet État peuplé de bourreaux et de victimes

que certains films se plaisent aujourd'hui à décrire.

Oui, un trop grand nombre de gens ont souffert de la RDA et lui ont tourné le dos.

Des centaines de milliers l'ont quittée,

des millions sont restés

- et pas seulement parce que le Mur était si haut.

Parmi ces derniers, beaucoup s'en vont maintenant seulement, en particulier des jeunes,

car ils ne trouvent plus de travail ni de perspective d'avenir ici.

Celui qui devait craindre la Sécurité d'État,

chaque fois qu'il franchissait les limites de ce qui était autorisé,

craint aujourd'hui souvent un danger bien pire,

celui qui pèse sur toute son existence sociale.

$\mathrm{Au}$ quotidien manque généralement la dimension de l'exceptionnel, du singulier, du significatif,

mais ce quotidien, c'est tout de même l'aspect essentiel de la vie d'un homme

et selon nous pour cette raison-même ce qui mérite le plus d'être transmis.

Au fur et à mesure que croît la distance historique

il prend une dimension spectaculaire.

Et surtout :

il concerne tout le monde, les autres comme nous.

Comment s'expliquer autrement l'intérêt grandissant

pour les biographies des « enfants de Golzow »?

Le quotidien scolaire

de ces élèves qui entraient à l'école primaire peu après la construction du Mur de Berlin devint au fil des décennies un quotidien de la vie des hommes.

Le film le conserve pour l'éternité comme dans l'ambre.

47 Nous - Barbara et moi -

souhaitons aux jeunes Allemands assez de curiosité

pour apprendre comment ont vécu leurs aïeux

et savoir ce que fut cette RDA, tant pour ses réussites que pour ses échecs.

Et nous souhaitons que leurs parents ne passent pas leur vie en RDA sous silence,

mais, si on a attiré leur attention

sur Les Enfants de Golzow,

qu'ils recommandent ce film à leurs enfants.

48 Je pense aujourd'hui que nous aurions dû

faire figurer à la fin de notre dernier film ces mots 
qui ont été écrits à une autre époque et en une autre occasion.

C'est encore une fois une citation de Bert Brecht.

Le titre de ce poème célèbre est

" Aux générations suivantes ».

On peut y lire ceci :

Les forces étaient faibles.

Le but était bien loin.

Il était nettement visible,

Et néanmoins quasiment impossible à atteindre pour moi.

C'est ainsi que s'écoula mon temps,

Celui qui m'était accordé sur terre.

Vous qui surgirez des flots

Qui nous ont engloutis,

Souvenez-vous,

Quand vous parlerez de nos faiblesses,

De l'époque ténébreuse à laquelle vous avez échappé.

[...]

Et pourtant nous savons :

Haïr la bassesse, cela aussi déforme les traits.

S'emporter contre l'injustice,

Cela aussi rend la voix rauque.

Las, nous qui voulions préparer le terrain à la bonté,

Nous n'avons pu nous-mêmes être bons.

Mais vous, quand enfin le temps sera venu

Où l'homme sera un soutien pour l'homme,

Souvenez-vous de nous avec indulgence.

Je vous remercie de votre attention.

\section{NOTES}

1. Le jeudi 15 septembre 2011 une version abrégée du film Lignes de vie (Lebensläufe, 1980-1981) avait été projetée devant la salle comble de Mon Ciné à Saint-Martin-d'Hères. Outre le prologue et l'épilogue, la sélection comprenait les films consacrés à Marieluise, Dieter et Ilona.

2. Albrecht Ludwig Berblinger (1774-1829), tailleur de la ville wurtembergeoise d'Ulm, échoua en 1811 dans sa tentative de voler au-dessus du Danube.

3. Jeu de mots : $m u ß$ (graphie actuelle : muss) est la $1^{\text {re }}$ ou $3^{\text {e }}$ personne du singulier du verbe müssen (devoir, être contraint, obligé de...) au présent de l'indicatif. 
AUTEURS

BARBARA JUNGE

Cinéaste

WINFRIED JUNGE

Cinéaste 